\title{
Identification and Upregulation of Galactose/N-acetylgalactosamine Macrophage Lectin in Rat Cardiac Allografts with Arteriosclerosis
}

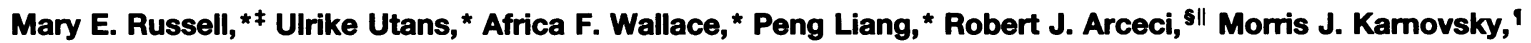 \\ Lauri R. Wyner," Yukari Yamashita, * and Chi Tarn* \\ *Cardiovascular Biology Laboratory, Harvard School of Public Health; ${ }^{\ddagger}$ Cardiovascular Division, Brigham and Women's Hospital; \\ ${ }^{8}$ Division of Pediatric Hematology-Oncology, Boston Children's Hospital; "Dana-Farber Cancer Institute; and 'Department of \\ Pathology, Harvard Medical School, Boston, Massachusetts 02115
}

\begin{abstract}
Using differential mRNA display to uncover potential mediators associated with chronic rejection, we identified a cDNA fragment induced in Lewis to F344 rat cardiac allografts with arteriosclerosis but not Lewis syngrafts. The full-length cDNA (1.4 kb) isolated from a rat cardiac allograft cDNA library was $99 \%$ identical to galactose $/ N$-acetylgalactosamine (Gal/GalNAc) macrophage lectin, a cell-surface receptor. This cDNA hybridized in Northern analysis with total RNA from eight cardiac allografts but not with host hearts, syngrafts, or other organs. There was a significant allograft-specific increase in transcript levels measured by reverse transcriptase $P C R$ at days $7,14,28$, and 75 in comparison with paired F344 host hearts (subject to same circulation but histologically normal), day-0 hearts, and syngrafts $(P<0.008, n=4$ at each time $)$. Transcript levels in cardiac allografts were higher than those in paired host spleens (a major source of inflammatory cells) ( $P$ $<0.0001$ ), indicating the localized nature of Gal/GalNAc lectin induction. By in situ hybridization and immunostaining, Gal/GalNAc lectin expression localized to a subset of inflammatory cells in cardiac allografts. These findings link Gal/GalNAc macrophage lectin to the chronic rejection process, as a possible mediator of macrophage infiltration. ( $J$. Clin. Invest. 1994. 94:722-730.) Key words: arteriosclerosis - asialoglycoproteins • cardiac transplantation • gene expression - rejection
\end{abstract}

\section{Introduction}

The major limitation to long-term survival after cardiac transplantation in humans is the development of chronic rejection (1). It is characterized by an obliterative arteriosclerosis with progressive neointimal thickening that eventually results in ischemic injury $(2,3)$. Studies of vessels from human heart transplant recipients have revealed an intimal hyperplasia that is concentric and diffuse, involves a spectrum of vessels, and is highly prevalent. The pathogenesis of transplant arteriosclerosis

Address correspondence to Dr. Mary E. Russell, Cardiovascular Biology Laboratory, Harvard School of Public Health, 677 Huntington Avenue, Boston, MA 02115.

Received for publication 26 October 1993 and in revised form 21 April 1994.

J. Clin. Invest.

(C) The American Society for Clinical Investigation, Inc. 0021-9738/94/08/0722/09 \$2.00

Volume 94, August 1994, 722-730 is unknown, and studies to elucidate the process have been limited by difficulty in obtaining useful clinical specimens: transplant arteriosclerosis occurs only in the donor heart and spares the host vessels. The leading hypothesis about the arteriosclerotic process holds that a chronic cell-mediated immune response to alloantigens produces cytokines that mediate neointimal smooth muscle cell accumulation in the graft-derived vasculature, in a manner analogous to the process of delayed-type hypersensitivity $(2,3)$.

Considerable insight into the cellular evolution of transplant arteriosclerosis has been gained recently from studies in a rat heterotopic abdominal cardiac transplantation model $(3,4)$. The combination of Lewis rat donors and F344 rat recipients results in long-term graft survival and a time-dependent development of arteriosclerotic lesions that resemble, on histologic examination, arteriosclerotic lesions in human transplant vessels $(3,4)$. Immunohistochemical studies with antibodies against monocytes, $\mathrm{T}$ cells, and smooth muscle cells have shown that arteriosclerotic lesions develop in three distinct stages $(5,6)$. In the first $30 \mathrm{~d}$, neointimal lesions are composed of infiltrating inflammatory cells rather than smooth muscle cells, which are predominantly macrophages with fewer lymphocytes. Between 45 and $90 \mathrm{~d}$ the infiltrating inflammatory cell population in the neointima decreases as intimal smooth muscle cells appear. In the last phase (beyond $90 \mathrm{~d}$ ), the neointima is maximally expanded, often obliterative, and composed predominantly of smooth muscle cells with fewer infiltrating mononuclear cells. The early and persistent presence of monocytes/macrophages in the first stage of arteriosclerosis suggests a prominent role for the macrophage in the initial phase of chronic rejection. To date there are few studies examining specific molecular mechanisms that may regulate the infiltration or function of macrophages in chronically rejecting hearts.

Previous studies from our laboratory have focused on the use of transcriptional analysis to study potential macrophage mediators in the Lewis to F344 rat chronic cardiac rejection model. We have used PCR-based methods because of their sensitivity and the small amount of tissue they require. We have found that IFN- $\gamma$ and monocyte chemoattractant protein-1 gene transcripts increase in cardiac allografts in a specific and localized fashion (7). ${ }^{1}$

However, chronic cardiac rejection is likely to be a complex process mediated by a spectrum of factors. As an alternative to transcriptional analysis of selected known factors that could be involved in chronic rejection, we have used a modified differential mRNA display technique (8) to identify potential mediators that are novel or have not been previously implicated in chronic

1. Russell, M. E., A. F. Wallace, N. E. S. Sibinga, L. R. Wyner, and M. J. Karnovsky, manuscript submitted for publication. 
rejection. In the present in vivo analysis of intact organs, we compared transcript patterns from Lewis to F344 cardiac allografts affected by chronic rejection with those of Lewis cardiac syngrafts exposed to the same surgical procedure but normal on histologic examination. These investigations identified a macrophage lectin cDNA fragment that was specifically induced in cardiac allografts. The purpose of this study was to clone the full-length cDNA and analyze its transcriptional induction patterns in chronic cardiac rejection.

\section{Methods}

Lewis to F344 rat cardiac transplantation. Heterotopic abdominal cardiac transplantation was performed as described with Lewis donor hearts (4) in an allogeneic combination involving F344 recipients. The syngeneic combination involved Lewis recipients and was performed to assess the contribution of surgical manipulation to the inflammatory response. Lewis hearts that had been harvested but not transplanted were used as reference groups matching the strain of donor or grafted hearts. At the time of harvest, both the host (recipient) and the transplanted hearts were collected for histologic analysis and RNA extraction. The host heart served as a reference that had been exposed to the same circulation but was normal on histologic examination. In some studies, the host spleen was also harvested for a comparison of transcription patterns in an organ rich in inflammatory cells but free of local allogeneic stimulation. In this study we used hearts harvested at 7 and $14 \mathrm{~d}$ because at these points macrophage infiltration starts to become evident. Midventricular sections were taken for histologic analysis at the time of harvest and snap frozen in liquid nitrogen for RNA extraction.

RNA isolation and Northern analysis. Total RNA was isolated with RNAzol B (Tel-test). Northern blots were prepared with $20 \mu \mathrm{g}$ of total RNA that had been electrophoresed through $1 \%$ agarose gels in the presence of $2.2 \mathrm{M}$ formaldehyde. Equivalent loading of samples was verified by ethidium bromide staining of the ribosomal bands. Capillary blotting was used for transfer to nylon nitrocellulose membranes (Micron Separation, Westboro, MA), which were then cross-linked under ultraviolet light (Stratagene, La Jolla, CA). Hybridization was completed with cDNA probes labeled with ${ }^{32} \mathrm{P}$ dCTP by random priming (Boehringer Mannheim Corp., Indianapolis, IN), and the blots were washed under high-stringency conditions $(0.2 \times \mathrm{SSC}, 0.1 \%$ SDS at $60^{\circ} \mathrm{C}$ ). Blots were exposed to PhosphorImager screens at $25^{\circ} \mathrm{C}$ or to Kodak X-Omat AR film with an intensifying screen at $-70^{\circ} \mathrm{C}$.

Differential mRNA display. Differential mRNA display analysis was performed as described ( 8 ) with the following modifications. We generated cDNA from six separate RNA populations representing two syngeneic hearts (normal on histologic examination) and four allografted hearts (with early signs of chronic cardiac rejection). Control studies included the substitution of water for cDNA or the omission of reverse transcriptase in the cDNA synthesis. PCR amplification with ${ }^{35}$ S-dATP was completed by using 5' primers that were arbitrary 10-mers (Kit A; Operon Technologies, Inc., Alameda, CA) and 3' primers that matched those used in the cDNA synthesis. All PCR reactions $(20 \mu \mathrm{l})$ were performed on a Perkin Elmer 9600 thermal cycler (Perkin-Elmer Corp., Norwalk, CT). Parameters for the display study included denaturation at $94^{\circ} \mathrm{C}$ for $15 \mathrm{~s}$, annealing at $40^{\circ} \mathrm{C}$ for $60 \mathrm{~s}$, and extension at $70^{\circ} \mathrm{C}$ for $20 \mathrm{~s}$ with a final 7 -min extension at the end of 40 cycles. The radioactive PCR amplification products were separated on 4 or $6 \%$ polyacrylamide gels. We defined differentially induced PCR amplification products as those that were present in all four allogeneic samples and absent in both syngeneic samples in at least four separate PCR experiments. Induced DNA was harvested from the sequencing gel by electroelution. To generate DNA for use in Northern blot hybridizations and for cloning, we reamplified the harvested DNA by subjecting it to an additional 40 PCR cycles in an 80- $\mu$ l reaction.

Cloning. Reamplified PCR products were directly cloned into the TA cloning vector PCR II (Invitrogen, San Diego, CA). The inserts were used as probes in Northern blot analysis of RNA from various cardiac allografts, syngrafts, and host hearts to confirm the allograftspecific hybridization pattern. The partial 3' cDNA fragment was then used to screen $\sim 500,000$ plaques from a bacteriophage lambda, custom Uni-ZAP cDNA library prepared from 14-d cardiac allograft poly (A) ${ }^{+}$ RNA (Stratagene, La Jolla, CA). Positive clones were isolated and rescued as plasmids, and their identity was verified by demonstrating allograft-specific hybridization on Northern blot analysis. DNA sequencing of both sense and anti-sense strands was performed with the Sequenase 2.0 kit (United States Biochemical Corp., Cleveland, $\mathrm{OH}$ ) on double-stranded plasmid DNA clones and subclones. Nucleotide and predicted amino acid sequence searches of the GenBank and EMBL data bases were performed with the GCG software package.

Semiquantitative reverse-transcriptase PCR assay. As an alternative to Northern blot analysis, we performed a semiquantitative reverse transcriptase PCR analysis to compare rat galactose/ $N$-acetylgalactosamine $(\mathrm{Gal} / \mathrm{GalNAc})^{2}$ macrophage lectin transcript levels. This allowed us to conserve RNA when samples were limited or evaluate samples such as isolated mononuclear cell types, which can yield $<10 \mu \mathrm{g}$ of total RNA. We modified a published reverse transcriptase PCR technique developed to measure differences in monocyte chemoattractant protein-1 transcript levels (7) for use with Gal/GalNAc macrophage lectin. cDNA synthesis was completed with random primers ( $2.5 \mu \mathrm{g}$ total RNA per reaction). Oligonucleotides were synthesized by Genosys Biotechnologies (The Woodlands, TX). The sequences were CCT AGA AAC CCT GAG AAC for the 5' primer and GAG TGC CGC TTA TTG TAG for the $3^{\prime}$ primer, chosen from the sequence analysis of our cDNA clone to result in a 941-bp product. Reaction conditions were as previously described. The thermal cycling parameters were denaturation at $94^{\circ} \mathrm{C}$ for $15 \mathrm{~s}$, annealing at $54^{\circ} \mathrm{C}$ for $20 \mathrm{~s}$, and extension for $60 \mathrm{~s}$ (with a final extension of $7 \mathrm{~min}$ at the end of all cycles). For semiquantitative PCR analyses, $150,000 \mathrm{cpm}$ of ${ }^{32} \mathrm{P}$-dCTP was included in the PCR reaction. The products were separated on $1 \%$ agarose gels which were dried and exposed to PhosphorImaging screens for $12 \mathrm{~h}$, and the amount of incorporated ${ }^{32} \mathrm{P}$ in amplified product bands was then measured by volume integration (Imagequant software; Molecular Dynamics Inc., Sunnyvale, CA).

To identify the optimum PCR conditions for accurate measurement of gene transcript levels, we established the logarithmic assay range with respect to cycle number and starting template concentration against different dilutions of cDNA. We used 30 cycles to perform the cDNA dilution study. Subsequent measurements of Gal/GalNAc macrophage lectin transcript levels were then completed at 30 cycles and a starting cDNA dilution of $1.25 \mu \mathrm{l}$. PCR amplification for 22 cycles with G3PDH, a ubiquitously expressed gene, was used as a control to assess variations in total RNA or cDNA loading between samples.' Corrected Gal/GalNAc macrophage lectin values were derived by dividing the measured amplified product value by the mean of the G3PDH value obtained for that cDNA from at least three analyses. PCR analyses were completed on each set of cDNAs at least four times. Results were subjected to MANOVA without replication. If a difference was significant, individual comparisons were made by the student's $t$ test, corrected by the Bonferroni method. Although there were variations in absolute values derived from different experiments, relative differences between cDNA sets analyzed at the same time were preserved.

Comparison of corrected Gal/GalNAc macrophage lectin levels. Differences in corrected Gal/GalNAc macrophage lectin transcript levels were examined in four separate studies. The first study was completed to compare differences in transcript levels at various points after cardiac transplantation. The $10 \mathrm{cDNAs}$ in this time-course study included samples from four cardiac allografts harvested 7, 14, 28, and 75 d after transplantation compared with one day- 0 Lewis heart, a total of three paired host hearts from days 7,14 , and 28 , and a day-14 Lewis syngraft with its paired host heart. The second cDNA analysis examined whether Gal/GalNAc macrophage lectin induction occurred systemi-

2. Abbreviation used in this paper: Gal/GalNAc, galactose/ $N$-acetylgalactosamine. 
cally or locally. cDNA levels in the host spleen (a major source of inflammatory cells) were compared with those in the allografted heart. The six cDNAs analyzed were derived from two additional allogeneic cardiac transplants: one harvested at $7 \mathrm{~d}$, the other at $28 \mathrm{~d}$. At each time point we compared the cDNAs from the host heart, allografted heart, and the host spleen. The third cDNA study examined Gal/GalNAc macrophage lectin gene expression in various populations of isolated rat inflammatory cells. Of the nine cDNAs studied, six were prepared from splenocytes (both adherent and nonadherent, each type stimulated with buffer, concanavalin A, and LPS/IFN- $\gamma$ ), two from separate thioglycolate-elicited macrophage preparations, and one from a 14-day cardiac allograft. The fourth cDNA study compared gene expression in cultured Lewis rat bone marrow-derived macrophages stimulated with isolated cytokines or a mixed culture containing lymphocytes and macrophages stimulated with concanavalin A.

Inflammatory cell populations. Isolated splenocytes were obtained by sieving splenic tissue into DME medium (GIBCO BRL, Gaithersburg, MD) as described (9). These cells were fractionated with FicollPaque (Pharmacia Fine Chemicals, Piscataway, NJ), and the mononuclear fraction was cultured at a density of $2-4 \times 10^{6}$ in a humidified incubator with 37 and $5 \% \mathrm{CO}_{2}$. A lymphocyte-enriched cell population was obtained by harvesting nonadherent cells by gentle washing $1 \mathrm{~h}$ after plating or by nylon wool purification. We judged the adherent cells to be phagocyte enriched by the fact that $20 \%$ of them stained with anti-macrophage antibody ED1. In contrast, few of the nonadherent cells were ED1 positive. Where indicated LPS $(100 \mathrm{ng} / \mathrm{ml}$; Sigma Chemical Co., St. Louis, MO), rat IFN- $\gamma(100 \mathrm{U} / \mathrm{ml}$; GIBCO-BRL $)$, concanavalin A ( $2 \mu \mathrm{g} / \mathrm{ml}$; Sigma Chemical Co.), or buffer alone was added to isolated cell populations and the cells were harvested $4 \mathrm{~h}$ later. Peritoneal inflammatory rat macrophages were elicited with thioglycolate medium as described (10). They were collected $4 \mathrm{~d}$ after induction of inflammation, separated on a Ficoll-Paque gradient, and plated at a density of 2 $\times 10^{6}$. At least $90 \%$ of the adherent cells were judged to be macrophages by morphologic criteria and ED1 staining.

Bone marrow-derived macrophages were obtained by culturing cells obtained from the femurs of Lewis rats in medium containing murine colony stimulating factor-1 (CSF-1) for 6-8 d as described (11). More than $95 \%$ of the cells stained positive for ED1 and were thus judged to be of a monocyte/macrophage lineage. Medium without CSF-1 was substituted for culture medium $24 \mathrm{~h}$ before stimulation with buffer, IFN$\gamma(100 \mathrm{U} / \mathrm{ml})$, murine IL-2 (10 U/ml; Genzyme Corp., Cambridge, MA), IL-4 (10 U/ml; Genzyme Corp.), IL-6 (20 U/ml; Genzyme Corp.), or concanavalin A $(2 \mu \mathrm{g} / \mathrm{ml})$. Mixed cultures were obtained by combining F344 lymphocytes (nylon wool-isolated splenocytes) activated with concanavalin A ( for $24 \mathrm{~h}$ ) directly with bone marrow-derived macrophage cultures treated with (or without) concanavalin A. The lymphocyte/macrophage ratios were 3:5 and 1:1. Lymphocytes alone activated with concanavalin A were used as a control. After $48 \mathrm{~h}$ we harvested the adherent cells and concentrated them (in a cytospin apparatus) onto microscope slides for immunohistochemical staining, or we extracted total RNA for reverse transcriptase PCR analysis. We assured the quality of the RNA before cDNA preparation by inspecting ribosomal RNA after $1 \mu \mathrm{g}$ had been separated on a 3-mm agarose gel.

In situ hybridization and immunohistochemistry. We completed in situ hybridization as described (12), using $5-\mu \mathrm{m}$ frozen sections obtained from 7-d cardiac allografts and paired host hearts. To generate radiolabeled antisense and sense transcripts, we linearized the full-length 1.4-bp bluescript cDNA and transcribed it with $\mathrm{T} 7$ or $\mathrm{T} 3$ polymerase using ${ }^{35} \mathrm{~S}$-UTP. The specificity of the antisense riboprobe was confirmed by hybridization in Northern analysis to 1.4-kb transcripts in lanes with cardiac allograft total RNA but not in lanes with day-0 heart total RNA. We identified cell types expressing the Gal/GalNAc lectin by immunohistochemical analysis of frozen sections $(4 \mu \mathrm{m})$ from 7 - and 28-day cardiac allografts, host spleens, and isolated rat cells prepared by the cytospin technique. A primary rabbit polyclonal antibody kindly provided by Dr. Toshisuke Kawasaki (13) was used to stain for Gal/ GalNAc macrophage lectin (crude IgG fraction at 1:500 dilution for $1.5 \mathrm{~h}$ at $25^{\circ} \mathrm{C}$ ) as previously described (7). Although the antibody was
A

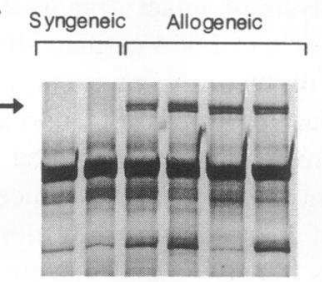

B

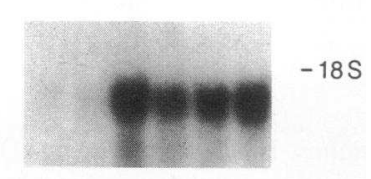

C

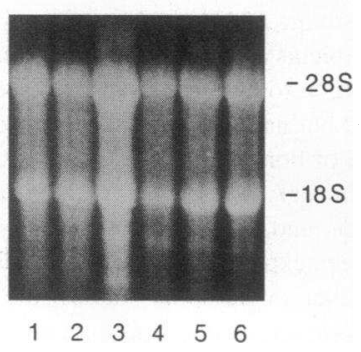

Figure 1. Differential mRNA display showing an upregulated PCR fragment in chronically rejecting hearts produced by allogeneic cardiac transplantation.

(A) The $6 \%$ polyacrylamide gel electrophoretic analysis of randomly amplified PCR products shows a cDNA fragment identified only in the four heart samples obtained after allogeneic transplantation (allografts) but not in the two hearts obtained after syngeneic transplantation (syngrafts). (B) The upregulated PCR fragment was harvested and reamplified from the differential display gel shown in $A$. When radiolabeled with ${ }^{32} \mathrm{P}$ and used as a probe in Northern analysis it hybridized to $1.4-\mathrm{kb}$ transcripts found only in the four lanes containing the hearts subjected to allogeneic transplantation, which develop

chronic rejection (lanes 3-6), but not to the two hearts from syngeneic cardiac transplantation (lanes 1 and 2). Samples from the same total RNA extraction were used in both the PCR and Northern analyses. $(C)$ RNA gel stained with ethidium bromide before transfer, to demonstrate loading in each lane of $20 \mu \mathrm{g}$ of total RNA.

raised against rat hepatic lectin, it does cross react with Gal/GalNAc macrophage lectin (13). Negative controls included omission of the primary antibody and use of another primary antibody. We also stained with antibody ED1 (Serotec Ltd., Oxford, UK), which recognizes monocytes/macrophages.

\section{Results}

Differential expression of a Gal/GalNAc macrophage lectin 3' fragment in allografts. We used differential display techniques to identify transcriptionally induced genes in a panel of cDNAs derived from syngeneic heart transplants in comparison with allogeneic heart transplants. Analysis of polyacrylamide gels containing randomly amplified PCR products obtained by using OPA 4 (AAT CGG GCT G) as a $5^{\prime}$ primer and T 12 VTC (where $\mathrm{V}$ included $\mathrm{A}, \mathrm{C}$, and $\mathrm{G}$ ) as a $3^{\prime}$ primer identified an $\sim 380$ bp fragment in sample lanes from allogeneic hearts but not in those from syngeneic hearts (Fig. $1 \mathrm{~A}$ ). When the cDNA in these upregulated bands was harvested, reamplified, and used to probe Northern blots, an allograft-specific hybridization pattern was visible (Fig. $1 \mathrm{~B}$ ). Transcripts of $1.4 \mathrm{~kb}$ were identified in lanes 3-6, containing total RNA derived from four hearts after allogeneic transplantation, but not in lanes 1 and 2, containing RNA from two syngeneic transplants. RNA loading prior to transfer is shown in the ethidium-stained agarose gel (Fig. 1 C). These findings confirmed the gene regulation pattern identified in the differential mRNA display analysis performed with the same panel of total RNAs. Northern analysis was then completed with total RNA obtained from an additional six allogeneic transplants (three each harvested at 7 and $14 \mathrm{~d}$ ) and two syngeneic transplants (both harvested at $14 \mathrm{~d}$ ). Fig. 2 shows a strong hybridization to $1.4-\mathrm{kb}$ transcripts in all six lanes (lanes 
A

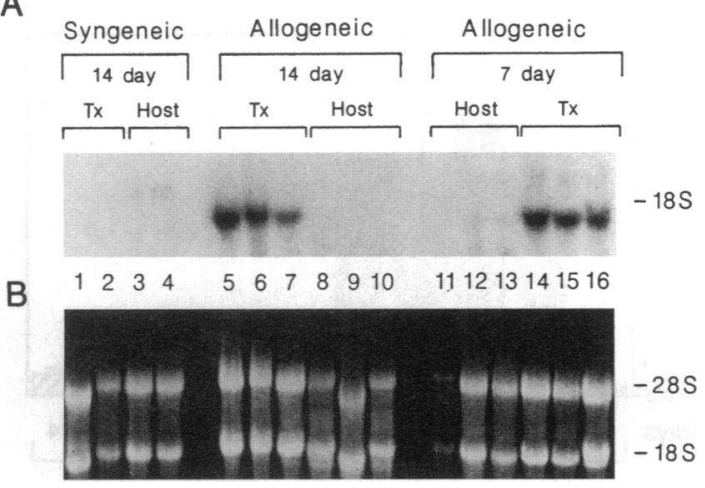

Figure 2. Upregulation occurring in an expanded series of 7- and 14day cardiac allografts but not in paired host hearts. Northern blot analysis using the transplanted heart (cardiac allograft) and host heart (exposed to the same circulation but histologically normal) from an additional two syngeneic and 6 allogeneic cardiac transplantations confirms and extends the allograft-specific induction patterns. $(A)$ The PCR-amplified DNA fragment from the differential display study hybridizes to $1.4-\mathrm{kb}$ transcripts found only in allogeneic transplanted hearts harvested at 7 and 14 days (lanes $5-7$ and 14-16) but not to transcripts from the paired host hearts or syngeneically transplanted hearts. $(B)$ RNA gel stained with ethidium bromide before transfer, to demonstrate loading in each lane of $20 \mu \mathrm{g}$ of total RNA (with the exception of lane 11).

5-7 and 14-16) containing allografted heart samples, in contrast with the lanes containing the six paired host-heart samples and the four syngeneic-heart samples (two hosts and two syngrafts). Taken together (Figs. 1 and 2), these findings indicate that the induction was not restricted to individual animals or procedures but occurred uniformly in Lewis to F344 cardiac transplantation.

Cloning of Gal/GalNAc macrophage lectin from a rat cardiac cDNA library. Direct cloning of the amplified PCR fragment harvested from the differential display gel produced a 380bp insert. When this insert was used as a probe in Northern blot analysis it hybridized to transcripts of 1.4-kb (data not shown) in lanes containing RNA from cardiac allografts but not in lanes containing samples from the host hearts, again reproducing the pattern identified by the differential display analysis. To determine the identity of the full-length cDNA, we screened a cardiac allograft cDNA library (Stratagene) with the 380-bp cloned PCR fragment. Nine recombinants were identified, the largest of which was $1.4 \mathrm{~kb}$. Sequence analysis of this fragment and homology searching revealed that the fragment was $99 \%$ identical to rat Gal/GalNAc macrophage lectin mRNA (GenBank accession number J05495) submitted by Ii et al. (14). The J05495 cDNA had been cloned from a rat peritoneal macrophage cDNA library.

Characterization of Gal/GalNAc macrophage lectin gene expression with a full-length cDNA clone. We examined the specificity of Gal/GalNAc macrophage lectin gene expression by Northern blot analysis in various rat organs, particularly those known to contain resident mononuclear cells. Probing with our full-length Gal/GalNAc macrophage lectin cDNA, we observed strong hybridization only in lane 2 containing RNA from the 28-day cardiac allograft (Fig. 3). We included the sample from the host spleen (lane 3) to determine whether Gal/GalNAc macrophage lectin transcripts were induced by a systemic effect on macrophages after transplantation. The sam-

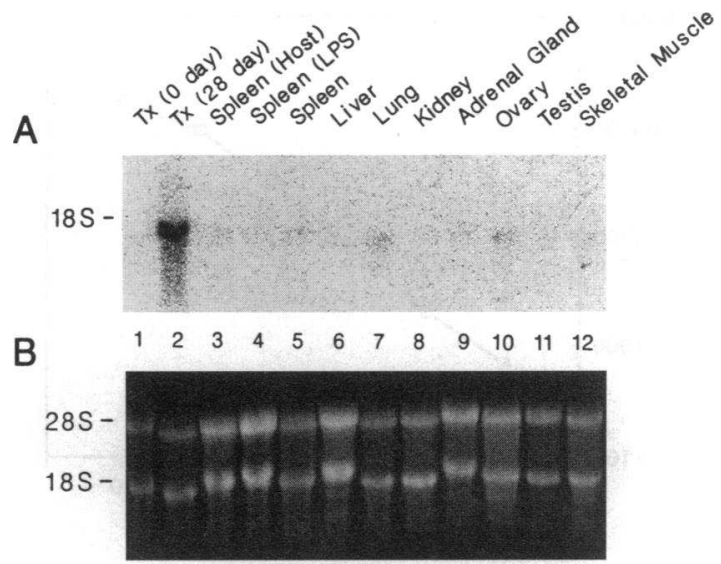

Figure 3. Organ blot analysis with the full-length Gal/GalNAc macrophage lectin cDNA showing tissue-specific upregulation in cardiac allografts. The full-length cDNA was isolated from a rat cardiac allograft cDNA library and used as a probe in Northern blot analysis completed with $20 \mu \mathrm{g} /$ lane total RNA from the indicated organs. $(A)$ Probe hybridizes strongly to 1.4-kb transcripts in the 28-day cardiac allograft sample. In contrast, hybridization levels were low in all other organs examined including those rich in resident monocytes and macrophages. To examine possible changes in expression related to systemic effects of inflammatory stimulation, the paired host spleen and a spleen harvested $8 \mathrm{~h}$ after intraperitoneal LPS injection were included. $(B)$ RNA gel stained with ethidium bromide before transfer, to demonstrate loading. Each lane contains $20 \mu \mathrm{g}$ of total RNA.

ple from the spleen of a rat $8 \mathrm{~h}$ after intraperitoneal treatment with LPS (lane 4) was included to see whether this potent inflammatory stimulus would alter Gal/GalNAc macrophage lectin expression in the spleen. A control spleen sample (lane 5) harvested without any stimulation was also included. All three samples from spleens showed faint to no hybridization, even though spleens are the principal source of macrophages. Similarly, hybridization signals were not apparent in the lanes containing RNA from other organs including the liver (lane 6), which contains Kupffer cells, lung (lane 7), which contains alveolar macrophages, kidney (lane 8), adrenal gland (lane 9), ovary (lane 10), testes (lane 11), and skeletal muscle (lane 12). This restricted pattern of Gal/GalNAc macrophage lectin expression suggests that its induction is specific to and localized within the cardiac allograft.

Reverse transcriptase PCR measurement of Gal/GalNAc macrophage lectin transcript levels. To have a more sensitive method of measuring Gal/GalNAc macrophage lectin transcripts, we developed a reverse transcriptase PCR assay using specific primers. We first determined the conditions at which the PCR amplification rate was logarithmic in order to avoid the amplification plateau at which the PCR product level is no longer proportional to the starting template level. Gal/GalNAc macrophage lectin gene amplification (Fig. 4) was linear over eight PCR cycles (upper panel) and by more than two logs in initial template or cDNA concentration (represented as the calculated amount of cDNA in the PCR reaction) (lower panel). These ranges are consistent with those typically found in PCR assay systems (15). For subsequent comparisons of Gal/GalNAc macrophage lectin gene transcript levels in various sets of cDNAs, we completed PCR amplification for the control gene, G3PDH, to derive corrected or normalized levels. This 

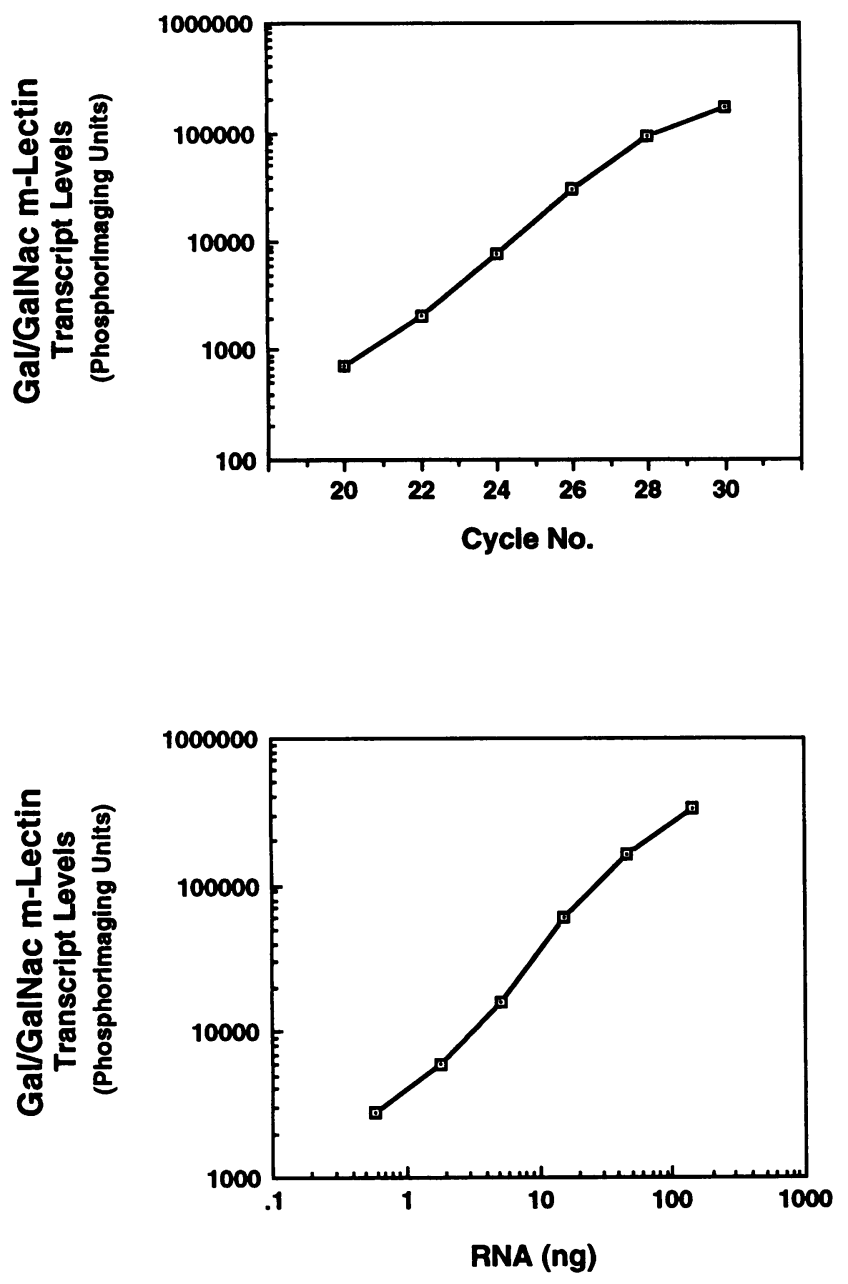

Figure 4. Linear range of Gal/GalNAc macrophage lectin reverse transcriptase PCR assay. Rat cardiac allograft cDNA was amplified using specific Gal/GalNAc macrophage lectin primers and separated electrophoretically on $1 \%$ agarose gels. ${ }^{32} \mathrm{P}$ incorporation in the PCR product band from dried gels was measured in PhosphorImager units. The linear relationship between amplified Gal/GalNAc macrophage lectin product bands and PCR cycle (upper panel) or added cDNA (represented as the calculated amount of total RNA in the PCR reaction, lower panel, using 30 cycles) identified the assay range in which amplified product was proportional to the initial target mRNA.

approach was used to compare relative differences between samples from three separate cDNA panels.

Specific and localized increase in corrected Gal/GalNAc macrophage lectin levels in cardiac allografts. Fig. 5 A shows that corrected Gal/GalNAc macrophage lectin gene transcript levels increased significantly in cardiac allografts (black bars) at all points studied (days 7, 14, 28, and 75) in comparison with the three reference groups: a day-0 heart (black bar), paired host hearts (hatched bars) and syngrafts (shaded bars) $(P<0.008)$. The increase in transcript levels between day 0 and day 28 measured by reverse transcriptase PCR (Fig. 5) correlated with the increase in hybridization measured by Northern blot analysis (Fig. 3, lane 2, RNA from a day-28 allograft). Differences between transcript levels at the various time points were not significant among allografts. This lack of a difference suggests that there was no further increase after the initial induc-
A

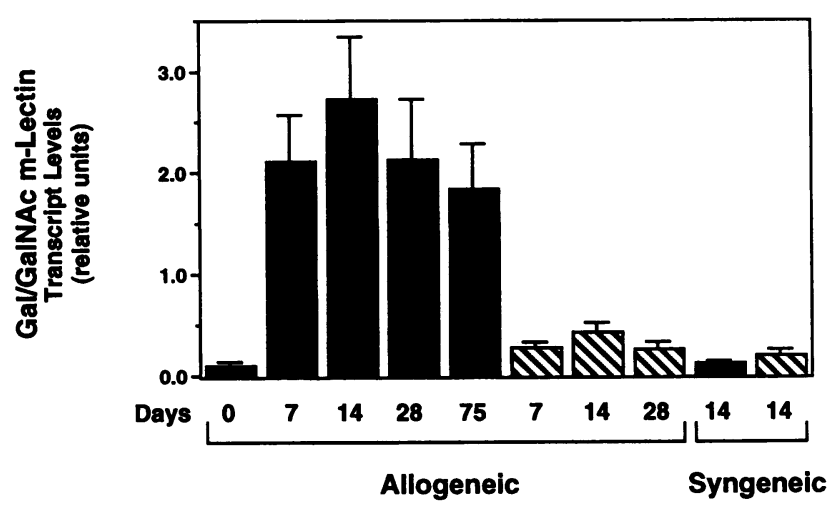

B

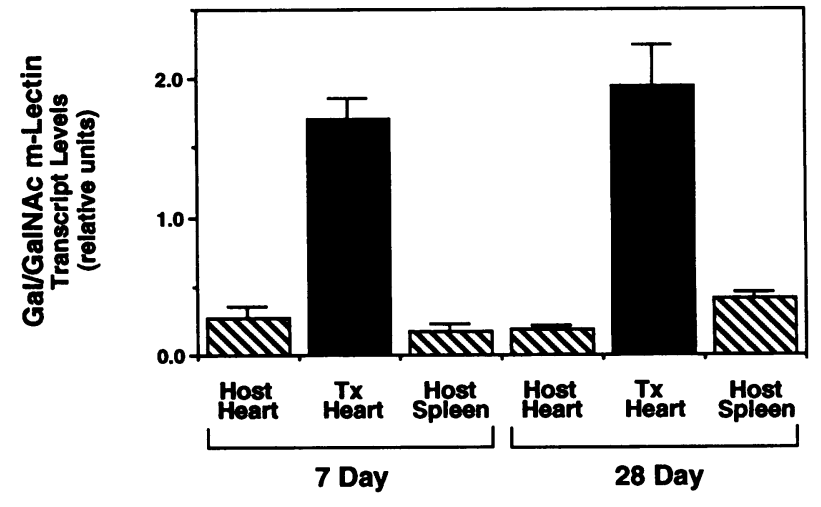

Figure 5. Time course and local nature of Gal/GalNAc macrophage lectin gene expression after allogeneic cardiac transplantation. Corrected levels were derived by normalizing Gal/GalNAc macrophage lectin reverse transcriptase $\mathrm{PCR}$ values after 30 cycles against those for the control gene, G3PDH, and are shown in relative units. $(A)$ There was a significant increase in cardiac transplant (or allograft) cDNA at 7, 14, 28, and 75 days (black bars) after transplantation compared with cDNA from the day- 0 heart (harvested but not transplanted), paired host hearts (hatched bars), and a 14-day syngraft (stippled bar) $(P<0.008)$. (B) $\mathrm{Gal} / \mathrm{GalNAc}$ macrophage lectin levels increased significantly in the transplanted hearts (black bars) at both 7 and 28 days compared with the host spleens (rich in resident macrophages but not subject to local allogeneic stimulation) and the host hearts normal on histologic examination $(P<0.0001)$, demonstrating the local nature of gene induction. Data are plotted as means \pm SEM and represent four separate PCR analyses.

tion, which would be consistent with an ongoing or chronic stimulation.

To examine whether Gal/GalNAc macrophage lectin gene induction was systemically or locally regulated, we compared levels in cardiac allografts with those in host spleens-reasoning that the spleen would be a major source of macrophages but be free of direct exposure to stimuli obtained in allografted tissue. As Fig. $5 B$ shows, Gal/GalNAc macrophage lectin transcript levels increased significantly in the 7-and 28-day cardiac allografts (black bars) relative to host spleens and hearts (hatched bars) $(P<0.0001)$. The low transcript levels in the host spleens suggest that Gal/GalNAc macrophage lectin gene 

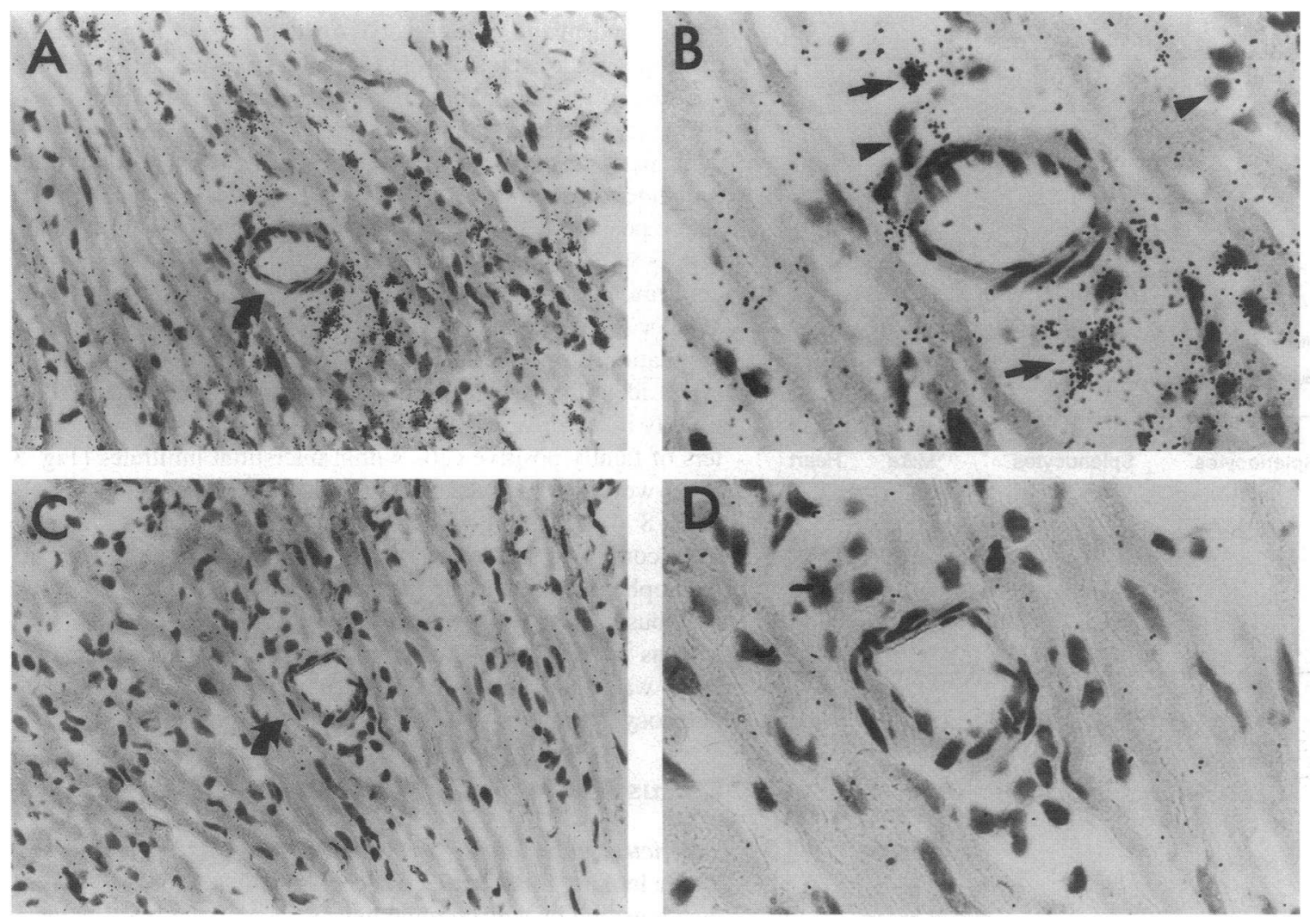

Figure 6. In situ localization of Gal/GalNAc macrophage lectin mRNA in cardiac allografts. Sections were hybridized to ${ }^{35} \mathrm{~S}$-labeled antisense $(A$ and $B$ ) or sense $(C$ and $D)$ riboprobes. Arrows in lower-power sections $(A$ and $C$ ) indicate the regions shown in higher magnification $(B$ and $D)$. Silver grains indicating hybridization of Gal/GalNAc macrophage lectin mRNA are clustered over a subset of inflammatory cells in the interstitium and perivascular spaces $(A, \times 260)$. Arrows mark representative positive mononuclear cells that are seen best at higher magnification $(B, \times 600)$. Arrowheads mark representative inflammatory cells without hybridization. Little hybridization in visible in adjacent noninflammatory cells such as cardiac myocytes. The sense sections show no significant hybridization $(C, \times 260)$ and $(D, \times 600)$.

induction in the cardiac allograft was due to local activation or stimulation.

Expression of Gal/GalNAc macrophage lectin mRNA in a subset of mononuclear cells in cardiac allografts. We performed in situ hybridization to localize the cell types expressing $\mathrm{Gal} /$ GalNAc macrophage lectin mRNA in cardiac allograft tissue. Positive hybridization to the antisense probe was visible in scattered mononuclear cells within inflammatory infiltrates in the interstitium and perivascular space (Fig. 6, $A$ and $B$ ), as evidenced by the clustering of silver grains (arrows). There was little hybridization to adjacent noninflammatory cells such as cardiac myocytes, or when the negative control, the sense RNA probe (Fig. 6, $C$ and $D$ ), was used in seriate sections. There was no significant hybridization of the antisense or sense probe to paired host hearts, which lacked inflammatory infiltrates (not shown). Previous immunohistochemical studies of such inflammatory infiltrates showed that they were composed predominantly of macrophages and a few T lymphocytes (6).

Corrected Gal/GalNAc macrophage lectin transcript levels in isolated inflammatory macrophages. To determine the specific inflammatory cell type expressing Gal/GalNAc macrophage lectin mRNA, we measured transcript levels in various isolated rat inflammatory cells. Fig. $7 \mathrm{~A}$ shows that corrected transcript levels increased significantly only in thioglycolateelicited macrophages and cells from a day-14 cardiac allograft $(P<0.0001)$ compared with adherent (macrophage-enriched) splenocytes (stimulated with buffer, concanavalin A, and LPS/ IFN- $\gamma$ ) and nonadherent (lymphocyte-enriched) splenocytes ( stimulated with buffer, concanavalin A, and LPS/IFN- $\gamma$ ). The identification of Gal/GalNAc macrophage lectin gene transcripts only in thioglycolate-elicited rat macrophages suggests that only certain conditions or certain macrophage subsets are associated with expression of this lectin.

We then used bone marrow-derived macrophages to identify inflammatory conditions that might upregulate lectin transcript levels in vitro. Fig. $7 \mathrm{~B}$ shows that Gal/GalNAc macrophage lectin transcripts were barely detectable in resting cells, as well as in those treated for $24 \mathrm{~h}$ with INF- $\gamma(100 \mathrm{U} / \mathrm{ml})$, LPS (100 $\mathrm{ng} / \mathrm{ml})$, IL-2 $(10 \mathrm{U} / \mathrm{ml})$, IL-4 $(10 \mathrm{U} / \mathrm{ml})$, and IL-6 (20 U/ $\mathrm{ml})$. We also found no induction of transcripts in additional studies completed with various doses of INF- $\gamma(1-1,000 \mathrm{U} /$ $\mathrm{ml})$ at various periods of incubation $(3-72 \mathrm{~h})$. However, we were able to confirm that the macrophages had been activated by the INF- $\gamma$ because of a fivefold increase in the level of nitrite released into the conditioned medium (measured by the Greiss reaction, which reflects nitric oxide production; data not shown). Because these isolated agents did not induce expression of bone marrow-derived Gal/GalNAc macrophage lectin, we studied the effect of mixed cultures of lymphocytes and macrophages. We used the mixtures as a way of stimulating the variety of cell types present in cardiac allografts and thioglycolate-elicited peritoneal inflammatory cells - two tissues in 
A

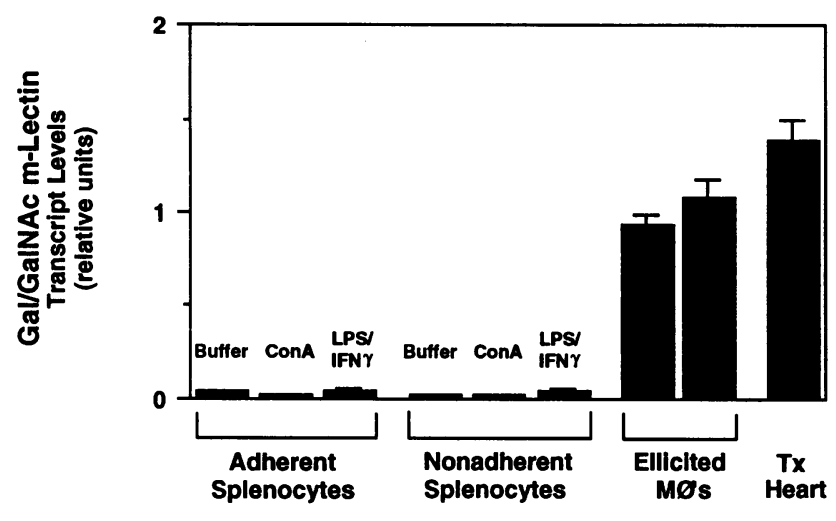

B

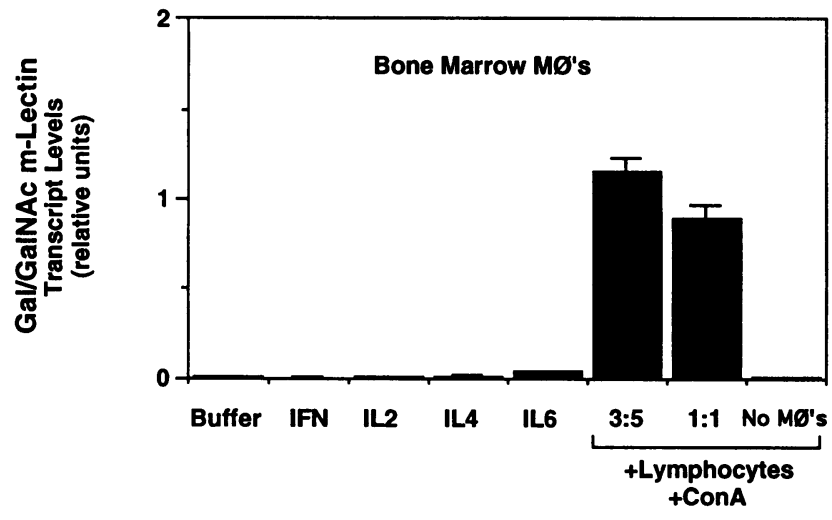

Figure 7. Increase in Gal/GalNAc macrophage lectin transcript levels in inflammatory macrophages. $(A)$ Corrected Gal/GalNAc lectin transcript levels were significantly higher in two separate thioglycolateelicited macrophage populations $(P<0.0001)$ and in cardiac allografts compared with adherent and nonadherent splenocytes even after $4 \mathrm{~h}$ of stimulation with concanavalin A or LPS/IFN- $\gamma$. (B) Gal/GalNAc macrophage lectin transcript levels are low in rat bone marrow-derived macrophages even after stimulation for $24 \mathrm{~h}$ with the listed cytokines. Levels increased significantly when macrophages were cultured in the presence of lymphocytes (at lymphocyte to macrophage ratios of 3:5 and $1: 1)$ and concanavalin $\mathrm{A}(P<0.0001)$. Data are plotted as means \pmSEM and represent four separate PCR analyses.

which lectin transcripts have been observed. Fig. $7 B$ shows that lectin transcript levels increased significantly $(P<0.0001)$ in the mixed cultures of F344 lymphocytes and Lewis macrophages at ratios of 3:5 and 1:1. We detected no lectin transcripts in lymphocytes alone after treatment with concanavalin A (Fig. $7 \mathrm{~B}$, right lane), in macrophages alone after treatment with concanavalin A (data not shown), or in mixed cultures of lymphocytes and macrophages not treated with concanavalin $A$ (data not shown). The results were similar in three separate mixed culture studies.

Identification of the Gal/GalNAc lectin protein. To identify cells expressing the lectin antigen or gene product, we used a polyclonal antibody directed against rat Gal/GalNAc hepatic lectin (which cross reacts with Gal/GalNAc macrophage lectin) to stain sections from resting bone marrow-derived macro- phages and mixed cultures of lymphocytes and bone marrow macrophages as well as cardiac allografts. There was no positive staining in resting bone marrow macrophages. However, positive cytoplasmic staining (Fig. $8 A$ ) was seen in $\sim 60 \%$ of the cells with macrophage morphology in the mixed cultures of lymphocytes and macrophages stimulated with concanavalin A. These positive cells were generally larger, had eccentric nuclei, and $>95 \%$ of them stained positive for ED1 (not shown), confirming that they were of a macrophage lineage. (The smaller ED1-negative cells likely represented the lymphocyte population.) Immunostaining of 7- and 28-day rat cardiac allografts identified a small subset of positive mononuclear cells, usually in the area of inflammatory infiltrates. There were clusters of faintly positive cells within interstitial infiltrates (Fig. 8 $B$ ) as well as occasional strongly positive cells in small vessels (Fig. $8 \mathrm{C}$ ). Staining with the monocyte-macrophage marker ED1 confirmed that many of the mononuclear cells were of macrophage lineage in the interstitium and vessel, as we showed previously (6). No positive staining was identified in host spleens or hearts or if primary antibody was omitted. Also, there was a less intense but diffuse cytoplasmic staining in hepatocytes in liver sections (not shown).

\section{Discussion}

Restricted upregulation of Gal/GalNAc macrophage lectin. While looking for potential molecular mediators of chronic cardiac rejection by a differential gene screening method, we identified a gene that proved to be Gal/GalNAc macrophage lectin. The gene transcripts we identified were specifically localized to and upregulated within Lewis to F344 rat cardiac allografts. This upregulation occurred early (by day 7) during initial macrophage accumulation and was sustained through days 14, 28, and 75 , as would be expected for a chronic inflammatory state characterized by ongoing macrophage infiltration. In contrast, transcript levels were low in three reference groups: paired host hearts (exposed to the same circulation but normal on histologic examination ), day-14 Lewis syngrafts (subject to the same surgical procedure but with matching host and recipient strains), and day- 0 Lewis hearts (harvested but not transplanted). Furthermore, the induction of Gal/GalNAc macrophage lectin mRNA occurred in a compartmental fashion restricted to the allografted tissue. Transcript elevation was not found in the matching host spleens (studied because they are a major source of macrophages but not subject to allogeneic stimulation), nor was it found in other organs rich in resident macrophages. This pattern is in keeping with the arteriosclerotic changes found in human cardiac transplants, which affect donor vessels but spare host vessels.

Using in situ hybridization and immunohistochemistry, we have demonstrated that a small subset of inflammatory cells (presumably macrophages) within the cardiac allograft express Gal/GalNAc macrophage lectin. In examining the specificity of Gal/GalNAc macrophage lectin gene expression in various isolated rat inflammatory cells in vitro, we found that this lectin was only present when macrophages were also exposed to thioglycolate-elicited lymphocytes or to lymphocytes in mixed culture with concanavalin A. Taken together, our in vivo and in vitro findings demonstrate that expression of Gal/GalNAc macrophage lectin was restricted to a specific population of inflammatory macrophages and suggest that this lectin is an inducible cell-surface receptor under tight regulatory control. 

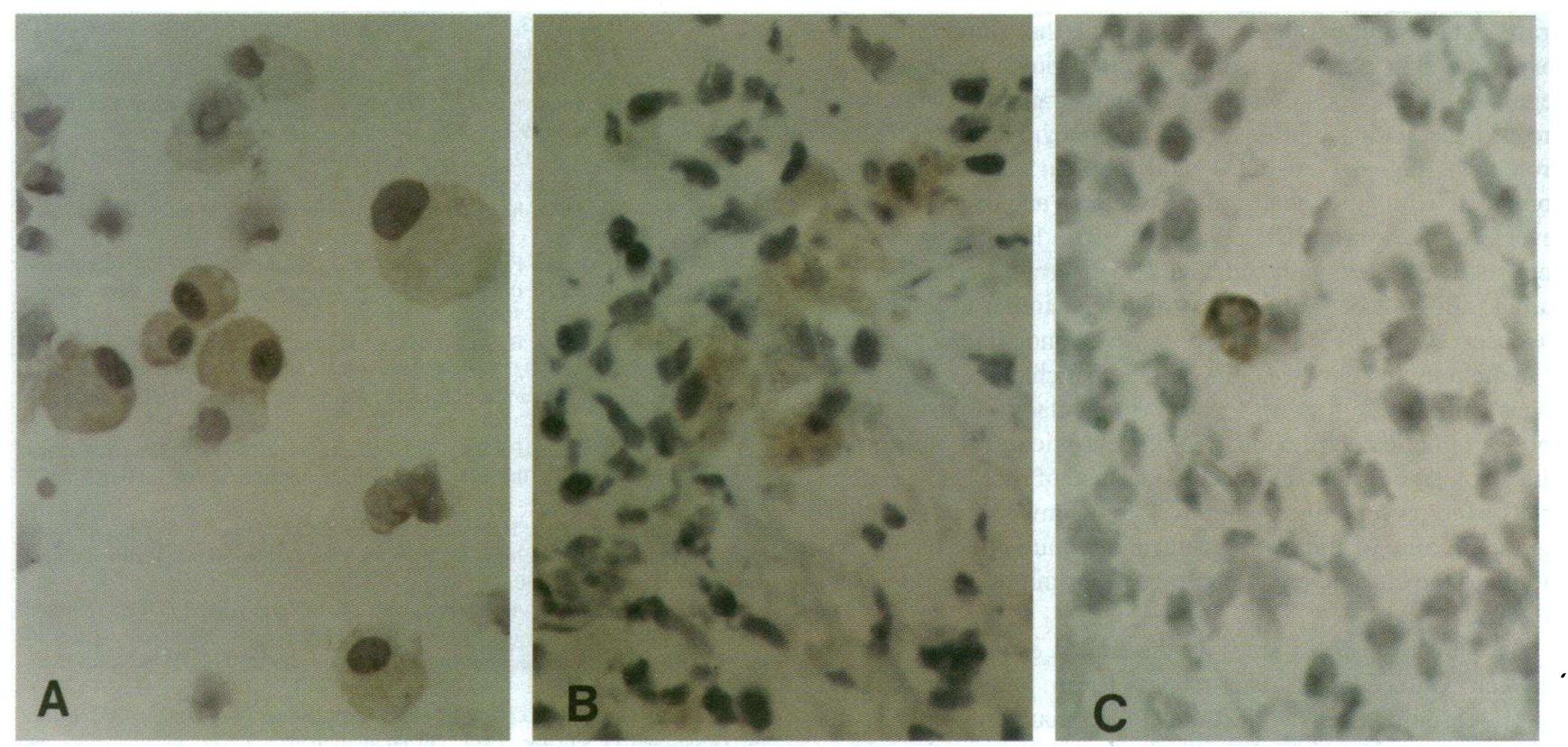

Figure 8. Expression of Gal/GalNAc lectin antigen measured by immunostaining with antibody against hepatic Gal/GalNAc lectin. (A) Cytospun bone marrow macrophages co-cultured with lymphocytes and concanavalin A showing many large positive cells of macrophage form. The small, negative cells are lymphocytes $(\times 360)$. $(B)$ Frozen section from a 28-day cardiac allograft showing a cluster of weakly positive mononuclear cells in a region of interstitial infiltration $(\times 320)$. $(C)$ Frozen section from a small vessel in a 28 -day cardiac allograft showing a strongly positive mononuclear cell $(\times 320)$.

Characteristics of Gal/GalNAc macrophage lectin. Lectins are a family of cell-surface proteins that specifically and selectively bind to complex carbohydrates on apposing cells (16). They have emerged as primary markers for cell recognition with clear functional roles. For example, interference with a lectin's binding to its apposing carbohydrate can disrupt bacterial and mononuclear cell attachment, tumor metastasis, and embryogenesis. Gal/GalNAc macrophage lectin falls into the category of C-type animal lectins characterized by calciumdependent activity and extracellular location. It has been studied independently by the laboratories of T. Osawa and T. Kawasaki, groups with divergent research interests. The Osawa group purified the murine lectin in an effort to identify the factor responsible for the tumor binding capacity it conferred to murine macrophages after stimulation with the antitumor streptococcal preparation OK-432 $(17,18)$. They showed increased lectin expression on the stimulated macrophages compared with resting ones. Antibody against the murine lectin prevented macrophage binding to and killing of tumor cells. The Kawasaki group cloned rat macrophage lectin, which they also designated macrophage-asialoglycoprotein-binding protein (m-ASGPBP), and performed comparisons with the extensively studied rat hepatic lectins (RHL) $(14,19,20)$. The Kawasaki group found that single-chain m-ASGP-BP forms homooligomeric receptors that bind and internalize ligand in a high-affinity fashion specific for Gal and GalNac (13). Rat hepatic lectin is an endocytic receptor for deglycosylated serum glycoproteins. The major form, RHL1, has $59 \%$ identity with the macrophage lectin cDNA. The two minor forms, RHL2 and -3, have $45 \%$ identity. An interesting variation in the macrophage lectin protein is the 24-amino acid insertion that includes an Arg-Gly-Asp (RGD) sequence. Because RGD is an integrin recognition sequence, it is possible that Gal/GalNAc macrophage lectin also confers integrin-mediated cellular adhesion.

Potential roles of Gal/GalNAc macrophage lectin. A spectrum of cell-surface molecules (or receptors) is thought to control macrophage function $(21,22)$. For example, integrin receptors such as $\mathrm{CD} 11 \mathrm{a} / \mathrm{CD} 18$ regulate integrin adhesion, and the mannose receptor (a macrophage lectin) is involved in endocytosis. Gal/GalNAc macrophage lectin may also fall into this receptor category given that it is specifically and locally upregulated in the context of a pathophysiologic process where the hallmark is monocyte/macrophage infiltration and arteriosclerosis. More studies are required to fully characterize factors upregulating lectin expression, to identify the functional role of Gal/ GalNAc macrophage lectin and to clarify the carbohydrate ligand on apposing (donor) cells. By analogy with other lectins, one can speculate that Gal/GalNAc macrophage lectin is involved in the recognition, adhesion, and infiltration of macrophages in cardiac allografts through exposed carbohydrates on the stimulated donor tissue.

From a clinical perspective, our observations in rat cardiac allografts may have pragmatic implications. For example, Gal/ GalNAc macrophage lectin transcript or protein levels in heart samples obtained by endomyocardial biopsy could serve as clinical markers of macrophage infiltration. Also, the development of inhibitors (peptides, antibodies, or carbohydrates) that block the lectin-carbohydrate interaction could provide a means of attenuating macrophage infiltration within allografts and disrupting the associated cytokine cascades believed to be initiated by macrophage activation.

Comparison of transcription patterns in Lewis to F344 cardiac allografts. The identification of Gal/GalNAc macrophage lectin as a gene whose transcripts are elevated in cardiac allo- 
grafts extends our earlier studies showing that some inflammatory factors in chronic cardiac rejection are transcriptionally regulated. In previous reports, we demonstrated persistently increased gene transcript levels for monocyte chemoattractant protein-1, IFN- $\gamma$, IL-6, CD4, and CD8 in cardiac allografts compared with host hearts, day- 0 hearts, and syngrafts by a reverse transcriptase PCR assay (7). ${ }^{1}$ The localized nature of gene induction seen with Gal/GalNAc macrophage lectin in the cardiac allograft but not the host spleen is similar to the pattern we observed with IFN- $\gamma$ and monocyte chemoattractant protein1. In the present study we demonstrate that changes in transcript levels can also be used to identify molecular factors that have not been implicated previously in the chronic rejection process. The advantage of this approach is our use of diseased hearts in an effort to preserve the pathophysiologic environment (including the mixture of infiltrating cells and stimuli) that could contribute to the chronic rejection process but might not be present in vitro.

The novelty of our study is the link it establishes in vivo between Gal/GalNAc macrophage lectin and a pathologic state-chronic cardiac rejection. Of particular interest is the unique localization of the expressed gene to a subset of mononuclear cells within the cardiac allograft. By also demonstrating that Gal/GalNAc macrophage lectin expression is restricted to a subpopulation of inflammatory macrophages in vitro, we further substantiate the theory that local activation of inflammatory cells plays a role in the phenomenon of chronic rejection. Taken together, our findings suggest that Gal/GalNAc macrophage lectin, a marker of inflammatory macrophages, may be one of the factors that mediate the recruitment or adhesion of macrophages in chronic cardiac rejection.

\section{Acknowledgments}

We are grateful to John David, Marla Steinbeck, and Laurie Hall for their support and advice on macrophage studies; Kirk Drickamer and Toshisuke Kawasaki for providing antibodies and helpful information; Edgar Haber for suggesting the differential display technique; Cesario Bianchi for photographic help; and Thomas McVarish for editorial assistance.

This work was supported in part by a grant from Bristol-Myers Squibb and by National Institutes of Health grant HL-43318.

\section{References}

1. Sharples, L. D., N. Caine, P. Mullins, J. P. Scott, E. Solis, T. A. English, S. R. Large, P. M. Schofield, and J. Wallwork. 1991. Risk factor analysis for the major hazards following heart transplantation-rejection, infection, and coronary occlusive disease. Transplantation. 52:244-252.

2. Schoen, F. J., and P. Libby. 1991. Cardiac transplant graft arteriosclerosis Trends Cardiovasc. Med. 1:216-223.
3. Cramer, D. V. 1993. Graft Arteriosclerosis in Heart Transplantation. R. G. Landes Company, Austin, TX. 1-68.

4. Adams, D. H., N. L. Tilney, J. J. Collins, and M. J. Karnovsky. 1992 Experimental graft arteriosclerosis. I. The Lewis-to-F-344 allograft model. Transplantation. 53:1115-1119.

5. Cramer, D. V., G. D. Wu, F. A. Chapman, E. Cajulis, H. K. Wang, and L. Makowka. 1992. Lymphocytic subsets and histopathologic changes associated with the development of heart transplant arteriosclerosis. J. Heart Lung Transplant. 11:458-466.

6. Adams, D. H., L. R. Wyner, and M. J. Karnovsky. 1993. Experimental graft arteriosclerosis. II. Immunocytochemical analysis of lesion development. Transplantation. 56:794-799.

7. Russell, M. E., D. H. Adams, L. R. Wyner, Y. Yamashita, N. J. Halnon, and M. J. Karnovsky. 1993. Early and persistent induction of monocyte chemoattractant protein 1 in rat cardiac allografts. Proc. Natl. Acad. Sci. USA. 90:60866090 .

8. Liang, P., and A. B. Pardee. 1992. Differential display of eukaryotic messenger RNA by means of the polymerase chain reaction. Science (Wash. DC). 257:967-971.

9. Stuart, A. E., J. A. Habeshaw, and A. E. Davidson. 1978. Phagocytes invitro. In Cellular Immunology. D. M. Weir, editor. Blackwell Scientific Publications, London. 31.1-31.30.

10. Steinbeck, M. J., A. U. Khan, and M. J. Karnovsky. 1993. Extracellular production of singlet oxygen by stimulated macrophages quantified using 9,10 diphenylanthracene and perylene in a polystyrene film. J. Biol. Chem. 268:1564915654.

11. Boltz-Nitulescu, G., C. Wiltschke, C. Holzinger, A. Fellinger, O. Scheiner, A. Gessl, and O. Förster. 1987. Differentiation of rat bone marrow cells into macrophages under the influence of mouse L929 cell supernatant. J. Leukocyte Biol. 41:83-91.

12. Arceci, R. J., A. A. J. King, M. C. Simon, S. H. Orkin, and D. B. Wilson. 1993. Mouse GATA-4: a retinoic acid-inducible GATA-binding transcription factor expressed in endodermally derived tissues and heart. Mol. Cell. Biol. 13:22352246.

13. Ozaki, K., M. Ii, N. Itoh, and T. Kawasaki. 1992. Expression of a functional asialoglycoprotein receptor through transfection of a cloned cDNA that encodes a macrophage lectin. J. Biol. Chem. 267:9229-9235.

14. Ii, M., H. Kurata, N. Itoh, I. Yamashina, and T. Kawasaki. 1990. Molecular cloning and sequence analysis of cDNA encoding the macrophage lectin specific for galactose and N-acetylgalactosamine. J. Biol. Chem. 265:11295-11298.

15. Ferre, F. 1992. Quantitative or semi-quantitative PCR: reality versus myth. In PCR Methods and Applications. Cold Spring Harbor Laboratory Press, Cold Spring Harbor, New York. 1-9.

16. Sharon, N., and H. Lis. 1989. Lectins as cell recognition molecules. Science (Wash. DC). 246:227-234.

17. Oda, S., M. Sato, S. Toyoshima, and T. Osawa. 1988. Purification and characterization of a lectin-like molecule specific for galactose/ $\mathrm{N}$-acetyl-galactosamine from tumoricidal macrophages. J. Biochem. (Tokyo). 104:600-605.

18. Oda, S., M. Sato, S. Toyoshima, and T. Osawa. 1989. Binding of activated macrophages to tumor cells through a macrophage lectin and its role in macrophage tumoricidal activity. J. Biochem. (Tokyo). 105:1040-1043.

19. Kawasaki, T., M. Ii, Y. Kozutsumi, and I. Yamashina. 1986. Isolation and characterization of a receptor lectin specific for galactose/ $\mathrm{N}$-acetylgalactosamine from macrophages. Carbohydr. Res. 151:197-206.

20. Ii, M., T. Kawasaki, and I. Yamashina. 1988. Structural similarity between the macrophage lectin specific for galactose/N-acetylgalactosamine and the hepatic asialoglycoprotein binding protein. Biochem. Biophys. Res. Commun. 155:720-725.

21. Valente, A. J., M. M. Rozek, E. A. Sprague, and C. J. Schwartz. 1992. Mechanisms in intimal monocyte-macrophage recruitment. A special role for monocyte chemotactic protein-1. Circulation. 86:III-20-III-25.

22. Auger, M. J., and J. A. Ross. 1992. Macrophage surface receptors. In The Macrophage. C. E. Lewis and J. O'D. McGee, editors. IRL Press, Oxford. 1625. 